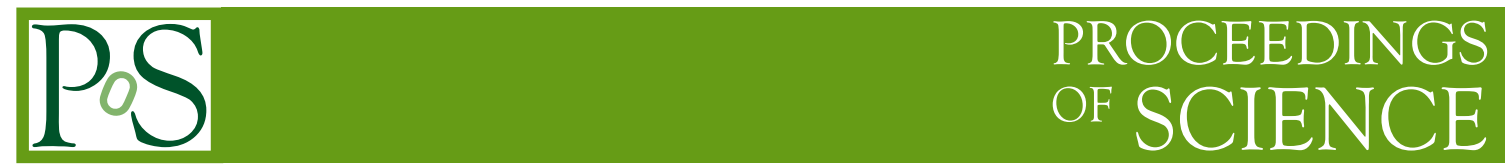

\title{
Notes on the New/Topologically Massive Gravity-like models beyond 3D
}

\author{
Yihao Yin* \\ Centre for Theoretical Physics, University of Groningen \\ E-mail: V.Vinerug.n]
}

\begin{abstract}
The New Massive Gravity and the Topologically Massive Gravity at the linearized level can be generalized to higher spin gauge theories in three dimensions. In this paper we discuss the possibility to further generalize them to higher dimensions. An example in seven dimensions will be shown in details.
\end{abstract}

Proceedings of the Corfu Summer Institute 2012 "School and Workshops on Elementary Particle Physics and Gravity"

September 8-27, 2012

Corfu, Greece

${ }^{*}$ Speaker. 


\section{Introduction}

The New Massive Gravity (NMG) [ [] and the Topologically Massive Gravity (TMG) [ [ $]$ ] are three dimensional massive gravity models with higher derivatives. In [四] and [四], it has been shown that the linearized NMG and TMG models can be extended to higher spin gauge theories in 3D. ${ }^{1}$

Starting from the spin-s Fierz-Pauli (FP) equations in 3D,

$$
\left(\square-m^{2}\right) \varphi_{\mu_{1} \cdots \mu_{s}}=0, \partial^{\mu_{1}} \varphi_{\mu_{1} \cdots \mu_{s}}=0, \eta^{\mu_{1} \mu_{2}} \varphi_{\mu_{1} \cdots \mu_{s}}=0,
$$

where $\varphi$ is a symmetric tensor, one can solve the divergenceless condition by

$$
\varphi_{\mu_{1} \cdots \mu_{s}}=G_{\mu_{1} \mu_{2} \cdots \mu_{s}}(h),
$$

where the $G(h)$ is a "generalized Einstein tensor", which is symmetric and contains $s$ derivatives:2

$$
G_{\mu_{1} \mu_{2} \cdots \mu_{s}}(h)=\varepsilon_{\mu_{1}}{ }^{v_{1} \rho_{1}} \cdots \varepsilon_{\mu_{s}}^{{ }^{v_{s}} \rho_{s}} \partial_{v_{1}} \cdots \partial_{v_{s}} h_{\rho_{1} \cdots \rho_{s}},
$$

and the gauge field $h$ is a rank-s symmetric tensor free of subsidiary constraints. Then by substituting the solution into the rest part of the FP equations, one can "boost up the deivatives", which leads to a spin-s NMG-like model: ${ }^{3}$

$$
\left(\square-m^{2}\right) G_{\mu_{1} \mu_{2} \cdots \mu_{s}}(h)=0, \eta^{\mu_{1} \mu_{2}} G_{\mu_{1} \mu_{2} \cdots \mu_{s}}(h)=0 .
$$

Due to the higher-derivative construction of the generalized Einstein tensor, this model possesses a gauge symmetry

$$
\delta h_{\rho_{1} \rho_{2} \cdots \rho_{s}}=\partial_{\left(\rho_{1}\right.} \xi_{\left.\rho_{2} \cdots \rho_{s}\right)},
$$

where the gauge parameter field $\xi$ is a symmetric tensor of rank $s-1$ free of subsidiary constraints.

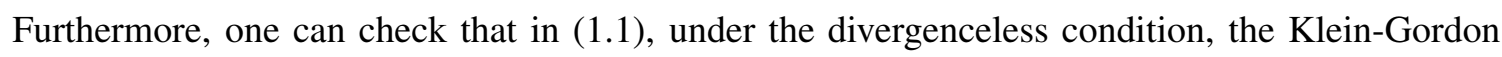
operator can be factorized into two first-order differential operators:

$$
\left(\varepsilon_{\mu_{1}}^{v \rho} \partial_{v} \pm m \delta_{\mu_{1}}^{\rho}\right)\left(\varepsilon_{\rho}^{\sigma \tau} \partial_{\sigma} \mp m \delta_{\rho}^{\tau}\right) \varphi_{\tau \mu_{2} \cdots \mu_{s}}=0
$$

By dropping the first operator, one obtains the so-called $\sqrt{\mathrm{FP}}$ equations

$$
\left(\varepsilon_{\mu_{1}} \sigma \tau \partial_{\sigma}-\mu \delta_{\mu_{1}}^{\tau}\right) \varphi_{\tau \mu_{2} \cdots \mu_{s}}=0
$$

where $\mu= \pm m$. One can check that $(\mathbb{L} . \mathbb{C})$ describes two propagating degrees of freedom, which are now separated into two opposite helicities, corresponding to the two choices of $\mu$. Then, again by "boosting up the derivatives", one obtains a spin-s TMG-like model ${ }^{4}$

$$
\left(\varepsilon_{\mu_{1}}^{\sigma \tau} \partial_{\sigma}-\mu \delta_{\mu_{1}}^{\tau}\right) G_{\tau \mu_{2} \cdots \mu_{s}}(h)=0
$$

with the same gauge symmetry as ([ए.5).

\footnotetext{
${ }^{1}$ In this paper, we only discuss free bosonic massive models on the flat background with the signature $(-,+,+, \cdots)$.

${ }^{2}$ One can check that for spin-2, $G(h)$ is the linearized Einstein tensor of the graviton (up to an overall factor).

${ }^{3}$ The spin-2 case is the linearized NMG.

${ }^{4}$ The spin- 2 case is the linearized TMG.
} 
In this paper, we discuss the possibility of extending the above 3D NMG/TMG-like models to higher dimensions. The discussion will be started in the context of the lower derivative theory in higher dimensions, then we will discuss the possibility of boosting up the derivatives, which may lead to NMG-like models. Afterwards we will further discuss the possibility of factorizing the Klein-Gordon operator in higher dimensions, which may lead to TMG-like models. After the general discussion, a concrete example in 7D will be presented.

\section{Tensors of Mixed Symmetry}

Before discussing higher dimensional models, the concept "spin" must be clarified. In general dimensions, the type of a particle is defined by its irreducible representation of the little group. In $3 \mathrm{D}$ or $4 \mathrm{D}$, where the little group for massive particles is $\mathrm{SO}(2)$ or $\mathrm{SO}(3)$, all inequivalent irreps can be represented by symmetric tensors of different ranks. Thus in 3D or 4D if we define "spin" to be the rank of the symmetric tensor, it is sufficient to label all different types of massive particles. However, for massive particles in $D \geq 5$, whose little group is $\mathrm{SO}(4)$ or larger, only one spin number is not sufficient to label all types of particles. In this situation, the irreps of the little group are represented by not only symmetric tensors, but also antisymmetric tensors and various mixed symmetry tensors. Consequently we need Young tableaux, instead of spin, to give each type of them a unique label. Nevertheless, we will still use the terminology "spin" to refer to the number of columns of a Young tableau.

When we talk about irreps of the little group, to be precise we are actually talking about traceless tensors with only spatial indices. However, the theories we would like to discuss are Lorentz covariant. Therefore, we need to Lorentz covariantize these tensors without changing the number of degrees of freedom they carry.

The FP theory has already told us how to Lorentz covariantize symmetric tensors. We conjecture that the same method applies also to other types of tensors:

(1) We replace all spatial indices with spacetime indices while still keeping their symmetry properties;

(2) Because we would like to go from the little group to the Lorentz group, its natural to modify the traceless condition by replacing the Kronecker delta $\delta_{i j}$ by the spacetime metric $\eta_{\mu v},{ }^{5}$

(3) Because the tensor now carries more components than the irrep originally had, some extra constraint is needed. We would like such a constraint to be Lorentz covariant, and we would like it to have no more than first-order derivatives, otherwise it might look like a dynamical equation rather than a constraint. Therefore, the only option seems to be the divergenceless condition, as already suggested by the FP theory.

This method definitely works for symmetric tensors as in the FP theory, and it also works for all types of tensors that are going to be discussed in this paper. To illustrate this, we count, as an example, the degrees of freedom of the simplest mixed symmetry tensor in 4D. Denote the irrep $\square$ of $\mathrm{SO}(3)$ by a tensor $T_{i j, k}$ which satisfies ${ }^{6}$

$$
T_{i j, k}=\mathscr{Y}_{[2,1]} T_{i j, k} \text { and } \delta^{j k} T_{i j, k}=0,
$$

\footnotetext{
${ }^{5}$ We denote spacetime indices by Greek letters and spatial indices by Latin ones.

${ }^{6}$ We use commas to separate different sets of antisymmetrized indices. Starting from Section $\square$, indices on a tensor which are not separated by commas are always understood as antisymmetrized ones.
} 
where $\mathscr{Y}$ is a Young symmetrizer whose subscript denotes the height of each column of the corresponding Young tableaux. ${ }^{7}$ One can check that $T_{i j, k}$ carries 5 independent components. ${ }^{8}$ Now we go to the group $\mathrm{SO}(1,3)$ by replacing all spatial indices with spacetime ones, and hence obtain the tensor $T_{\mu v, \rho}$, which satisfies

$$
T_{\mu v, \rho}=\mathscr{Y}_{[2,1]} T_{\mu v, \rho} \text { and } \eta^{v \rho} T_{\mu v, \rho}=0
$$

carrying 16 independent components. We would like to impose the divergenceless condition ${ }^{9}$

$$
\partial^{\mu} T_{\mu v, \rho}=0
$$

in order to lower the degrees of freedom. One can check that $\partial^{\mu} T_{\mu v, \rho}$ is a non-symmetric traceless tensor, so it appears that (2.3]) gives $4 \times 4-1=15$ independent constraints, but this is an over-counting, because the double divergence on the antisymmetric pair of indices vanishes by construction

$$
\partial^{\mu} \partial^{v} T_{\mu v, \rho} \equiv 0
$$

Therefore we must compensate 4 degrees of freedom. Thus the total number of degrees of freedom of $T_{\mu v, \rho}$ is $16-15+4=5$, which is exactly the same number as that of $T_{i j, k}$.

Suppose such kind of counting always works, then we can write down the generalized version of FP equations

$$
\left\{\begin{array}{rl}
\left(\square-m^{2}\right) T_{\ldots} & =0 \\
\partial \cdot T_{\ldots} & =0 \text { (on all indices) } \\
\eta^{*} T_{\ldots} & =0 \text { (on all pairs of indices) }
\end{array},\right.
$$

where $T$ is a tensor of any allowed Young symmetry, and we use the Klein-Gordon equation to describe the free propagation. The divergenceless condition is always imposed on all indices and the traceless condition is always imposed on all pairs of indices. In the next section, we will discuss whether we can construct NMG-like models that are equivalent to this set of generalized FP equations, like what we did in 3D.

\section{Boosting Up the Derivatives}

In 3D we derived the NMG-like models by solving the divergenceless condition in the FP equations using the generalized Einstein tensor, and we wonder whether a similar way of boosting up derivatives exists in higher dimensions.

\footnotetext{
${ }^{7}$ We adopt this notation from [回]. In our definition, a Young symmetrizer is a projection operator acting on a multi-form in such a way that, after filling each set of antisymmetrized indices into the corresponding column of the Young tableau, it first symmetrizes indices on each row and then antisymmetrizes indices on each column. A Young symmetrizer is by definition normalized, i.e. $\mathscr{Y}^{2}=\mathscr{Y}$.

${ }^{8}$ For the little group SO(3), a (reducible) traceful tensor of the type $\square$ has 8 independent components. The trace of it, which is the fundamental representation $\square$, has 3 independent components. Then a traceless tensor of the type $\square$ has $8-3=5$ independent components. One can do a similar counting that the same type of traceless tensor in the Lorentz group $\mathrm{SO}(1,3)$ carries 16 independent components.

${ }^{9}$ Note that because of the Young symmetry, the tensor satisfies $T_{[\mu \nu, \rho]}=0$. Therefore, one can derive $\partial^{\rho} T_{\mu v, \rho}=0$ from $([2.3])$, i.e. the divergence hitting on any one of the three indices gives zero.
} 
Let us recall how we define the 3D generalized Einstein tensor $(\mathbb{L} .3)$. As shown in the following diagram, roughly speaking, it is defined in such a way that we first take the exterior derivative on every column of the Young tableau of the gauge field, resulting into the generalized Riemann tensor, ${ }^{10}$ and then take the Hodge duality on every column of the Riemann tensor, which leads to what we call the generalized Einstein tensor.

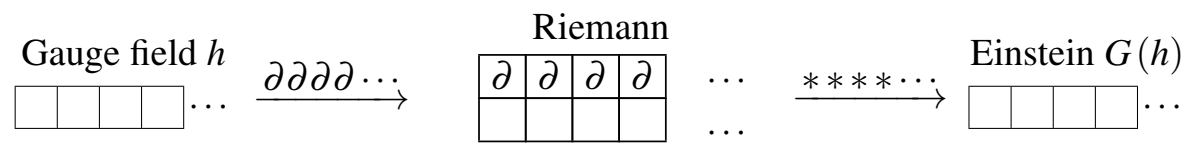

Note that there are two important facts in this way of construction.

First, in 3D, because the generalized Einstein tensor $G(h)$ has been constructed as the dual of the generalized Riemann tensor, setting $G(h)$ to zero means $h$ is a pure gauge, which does not describe any propagating degrees of freedom. In this way $G(h)=0$ gives only a trivial solution to the NMG-like equations of motion. This is important because otherwise the model would include massless propagating modes, and thus the model would not be equivalent to the FP theory.

Second, in 3D the generalized Einstein tensor $G(h)$ always lives in the same representation as the gauge field $h$ (off-shell traceful and symmetric rank-s tensors), otherwise it would be difficult to integrate the equations of motion into an action. ${ }^{11}$

In order to find direct extensions of the 3D NMG-like models in higher dimensions, we must be able to construct the generalized Einstein tensor with both properties mentioned above in $D>3$. However, it is difficult in general. For instance, in 4D, if we use a totally symmetric gauge field and define the generalized Einstein tensor as the dual of the generalized Riemann tensor, then as shown in the diagram

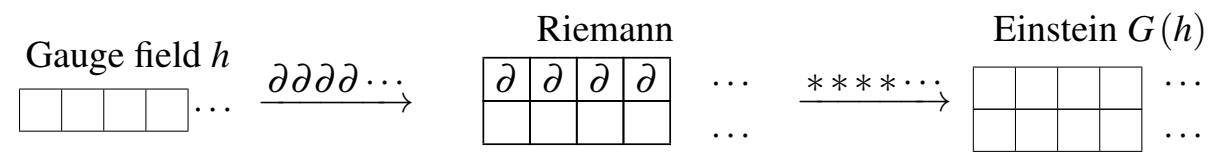

$G(h)$ no longer lives in the same representation as $h$. On the other hand, if we define the generalized Einstein tensor in another way that it lives in the same representation, ${ }^{12}$ then it carries less number of independent components than the generalized Riemann tensor, which means $G(h)=0$ contains massless modes.

Only for gauge fields of specific representations (usually tensors of mixed symmetry) in specific dimensions can we define the generalized Einstein tensor to be the dual of the generalized Riemann tensor as well as in the same representation as the gauge field. Only in these situations, can we find a direct generalization of the 3D NMG-like models. In these situations, we can use the

\footnotetext{
${ }^{10}$ One can check that for spin-2, the linearized Riemann tensor can be written as (up to an overall factor) taking an exterior derivative on each of the two indices of the graviton. If the generalized Riemann tensor vanishes, then the gauge field must be a pure gauge.

${ }^{11}$ To get an intuitive idea, one can think that the simplest way to encode the equation $\left(\square-m^{2}\right) G \ldots(h)=0$ into a Lagrangian is to use the term $h^{\cdots}\left(\square-m^{2}\right) G \ldots(h)$, which requires that $h^{\cdots}$ and $G \ldots(h)$ have the same number of indices with the same symmetry property.

${ }^{12} \mathrm{See}$, for instance, the definition of the generalized Einstein tensor in [回], where it is basically constructed out of the traces of the Riemann tensor.
} 
generalized Einstein tensor to solve the divergenceless condition in (ㅍ.5) $:^{13}$

$$
T_{\ldots}=G_{\ldots}(h),
$$

where $T, G$ and $h$ have the same symmetry on their indices, and then substitute the solution into the other two equations in (2.5T), in order to obtain the higher derivative model

$$
\begin{aligned}
\left(\square-m^{2}\right) G_{\ldots}(h) & =0, \\
\eta{ }^{*} G_{\ldots}(h) & =0,
\end{aligned}
$$

which is the NMG-like model in higher dimensions.

Similar to 3D, in higher dimensions the NMG-like models also have gauge symmetries. Note that if we replace the gauge field with the derivative of any other field, because by definition the generalized Riemann tensor has already got a row of derivatives in its Young tableau, inevitably it will then acquire a pair of antisymmetrized derivatives, which gives zero. In this way, one can see that the gauge transformation rules are always parameterized by tensors with one index fewer than the gauge field has, and the missing index is carried by a derivative.

Now we discuss in details exactly what representations are allowed to construct NMG-like models in higher dimensions. The situations for spin-1, spin-2 and higher spins differ from each other. In the following they will be discussed separately.

Spin-1

Considering the criterion that the dual of the Riemann tensor has to live in the same representation as the gauge field, for spin- 1 the only allowed type of gauge field is represented by a single-column Young tableau of height $\frac{1}{2}(D-1)$ in an odd spacetime dimension $D$, as indicated in the diagram:

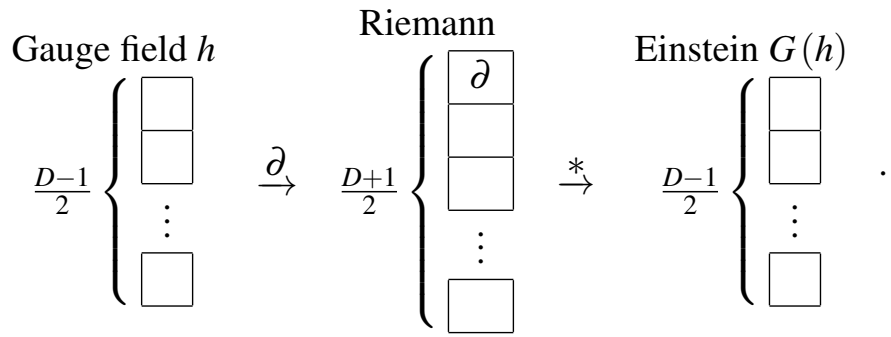

Starting from the generalized FP equations for spin-1 (or, the higher-rank generalization of the Proca model)

$$
\left(\square-m^{2}\right) T_{\mu_{1} \cdots \mu_{(D-1) / 2}}=0, \quad \partial^{\mu_{1}} T_{\mu_{1} \cdots \mu_{(D-1) / 2}}=0,
$$

where $T$ is a totally antisymmetric tensor, one can then solve the divergenceless condition

$$
T_{\mu_{1} \cdots \mu_{(D-1) / 2}}=G_{\mu_{1} \cdots \mu_{(D-1) / 2}}(h)=\varepsilon_{\mu_{1} \cdots \mu_{(D-1) / 2}} \mu_{(D+1) / 2} \cdots \mu_{D} \partial_{\mu_{(D+1) / 2}} h_{\mu_{(D+3) / 2} \cdots \mu_{D}},
$$

and substitute the solution into the Klein-Gordon equation to derive the NMG-like model:

$$
\left(\square-m^{2}\right) G_{\mu_{1} \cdots \mu_{(D-1) / 2}}(h)=0 .
$$

\footnotetext{
${ }^{13}$ The fact that the divergenceless condition can be solved in this way is a generalization of the Poincaré lemma [0].
} 
Obviously this model has the gauge symmetry

$$
\delta h_{\mu_{1} \cdots \mu_{(D-1) / 2}}=\partial_{\left[\mu_{1}\right.} \xi_{\left.\mu_{2} \cdots \mu_{(D-1) / 2}\right]} .
$$

Note that not all components of the gauge parameter field contribute to the transformation, i.e. there is a "gauge transformation" of the gauge parameter field itself: $\delta \xi_{\mu_{2} \cdots \mu_{(D-1) / 2}}=\partial_{\left[\mu_{2}\right.} \zeta_{\left.\mu_{3} \cdots \mu_{(D-1) / 2}\right]}$. This must be taken care of when we count the degrees of freedom and when we do the gauge-fixing. This also matters for spin-2 and higher spins. We will not further discuss it in this section, but in Section [1, for a specific 7D spin-2 example we will show some details.

In $D=4 k-1$, where $k=1,2, \cdots$, , (B.5) can be easily integrated into an action

$$
S_{\mathrm{NMG} \mathscr{Y}_{[2 k-1]}}=\int d^{4 k-1} x h^{\mu_{1} \cdots \mu_{2 k-1}}\left(\square-m^{2}\right) G_{\mu_{1} \cdots \mu_{2 k-1}}(h),
$$

but unfortunately in $D=4 k+1$, we have not yet found an action, because in this situation one can prove that the Klein-Gordon term $h^{\mu_{1} \cdots \mu_{2 k}}\left(\square-m^{2}\right) G_{\mu_{1} \cdots \mu_{2 k}}(h)$ is actually a total derivative.

\section{Spin-2}

For spin-2 in $D$ dimensions, the allowed types of tensors always carry in total $D-1$ indices. As shown in the following diagram, denoting $p$ and $q(p \geq q)$ as the heights of the first and second columns of the Young tableau of the gauge field, if $p+q=D-1$, then after taking exterior derivatives and Hodge dualities, the height- $p$ column ends up in a height- $q$ column and vice versa.

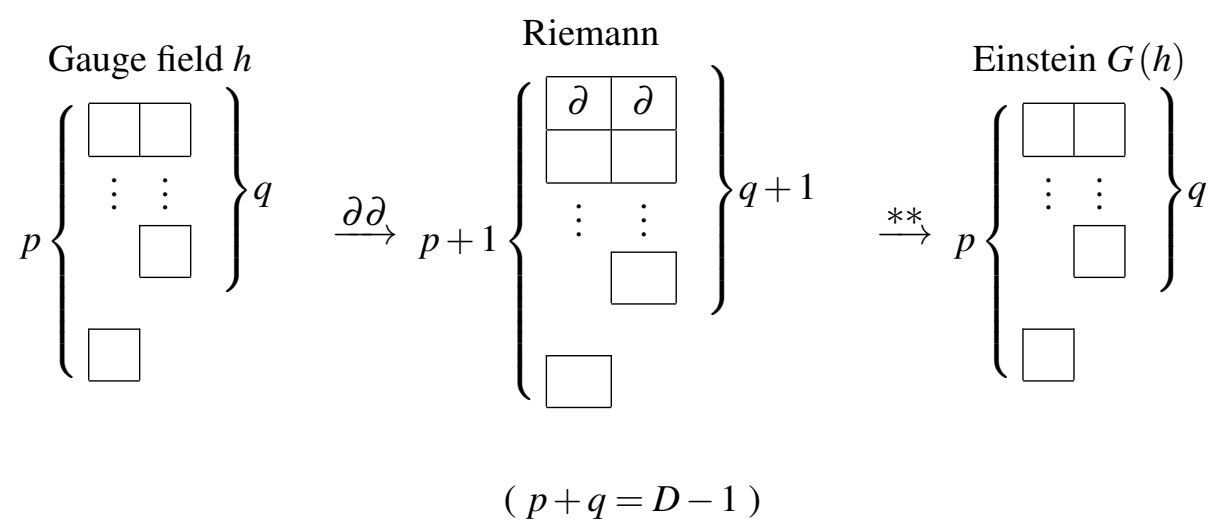

To write explicitly, the generalized Einstein tensor is constructed as

$$
G_{\mu_{1} \cdots \mu_{p}, v_{1} \cdots v_{q}}(h)=\varepsilon_{v_{1} \cdots v_{q}}^{\alpha \rho_{1} \cdots \rho_{p}} \varepsilon_{\mu_{1} \cdots \mu_{p}}^{\beta \sigma_{1} \cdots \sigma_{q}} \partial_{\alpha} \partial_{\beta} h_{\rho_{1} \cdots \rho_{p}, \sigma_{1} \cdots \sigma_{q}}
$$

Then in the spin-2 generalized FP equations ${ }^{14}$

$$
\left(\square-m^{2}\right) T_{\mu_{1} \cdots \mu_{p}, v_{1} \cdots v_{q}}=0, \quad \partial^{\mu_{1}} T_{\mu_{1} \cdots \mu_{p}, v_{1} \cdots v_{q}}=0, \quad \eta^{\mu_{1} v_{1}} T_{\mu_{1} \cdots \mu_{p}, v_{1} \cdots v_{q}}=0,
$$

one can solve the divergenceless condition by

$$
T_{\mu_{1} \cdots \mu_{p}, v_{1} \cdots v_{q}}=G_{\mu_{1} \cdots \mu_{p}, v_{1} \cdots v_{q}}(h),
$$

\footnotetext{
${ }^{14} T$ satisfies the Young symmetry $\mathscr{Y}_{[p, q]}$. Consequently, $T_{\left[\mu_{1} \cdots \mu_{p}, v_{1}\right] \cdots v_{q}}=0$, which means $\partial^{\mu_{1}} T_{\mu_{1} \cdots \mu_{p}, v_{1} \cdots v_{q}}=0$ implies that $\partial^{v_{1}} T_{\mu_{1} \cdots \mu_{p}, v_{1} \cdots v_{q}}=0$.
} 
which leads to the NMG-like model

$$
\left(\square-m^{2}\right) G_{\mu_{1} \cdots \mu_{p}, v_{1} \cdots v_{q}}(h)=0, \quad \eta^{\mu_{1} v_{1}} G_{\mu_{1} \cdots \mu_{p}, v_{1} \cdots v_{q}}(h)=0 .
$$

This model has the gauge symmetry

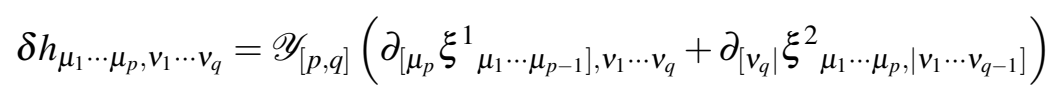

for $p>q$, where $\xi^{1}$ and $\xi^{2}$ satisfy the Young symmetries $\mathscr{Y}_{[p-1, q]}$ and $\mathscr{Y}_{[p, q-1]}$, respectively. For $p=q$, because the two columns of the Young tableau of $h$ are symmetric, using one tensor $\xi$ of the type $\mathscr{Y}_{[p, p-1]}$ is sufficient to parameterize the transformation:

$$
\delta h_{\mu_{1} \cdots \mu_{p}, v_{1} \cdots v_{p}}=\mathscr{Y}_{[p, p]}\left(\partial_{\left[v_{p} \mid\right.} \xi_{\left.\mu_{1} \cdots \mu_{p}, \mid v_{1} \cdots v_{p-1}\right]}\right) .
$$

We have not yet studied in general the possibility to integrate (3.TI) into an action, but two specific examples have been studied in details. One example is the gauge field of the type $\square$ in 4D. A NMG-like ghost-free action carrying 5 massive propagating degrees of freedom has been constructed using this type of gauge field in $[\square]$. The other example is $\square$ in $7 \mathrm{D}$, which is going to be discussed in Section [5.

\section{Higher spins}

For spin-3 or higher, besides the criterion that the dual of the Riemann tensor and the gauge field should have the same symmetry, there is another criterion which further eliminates a lot of representations: there should not be more than $D-1$ boxes in the first two columns of the Young tableau of the gauge field. ${ }^{15}$ Then under these two criteria, one can conclude that the only allowed types of gauge fields are represented by rectangular Young tableaux of height $\frac{1}{2}(D-1)$ :

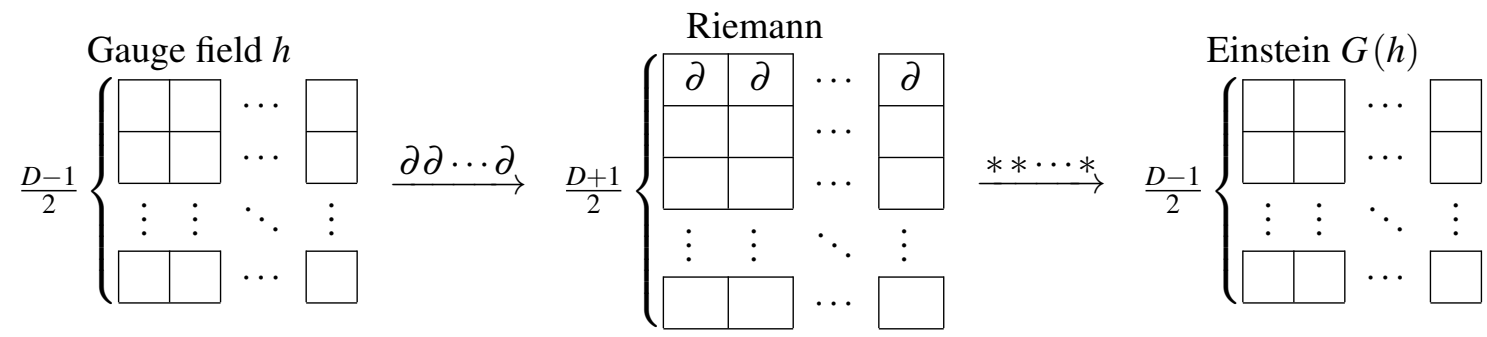

As one can see, the first two columns of $h$ 's Young tableau contain already $D-1$ boxes, which is the maximal number allowed by the second criterion mentioned above. Furthermore, the first criterion implies that if we took away one box from a column, we had to put this box back into another column (just like the spin- 2 case, this pair of columns should add up to $D-1$ boxes), and thus the total number of boxes in the two longest columns had to exceed the limit $D-1$. This is the reason why there are no allowed types of Young tableaux other than rectangular ones.

Again, one may solve the divergenceless condition in the generalized FP equations using the generalized Einstein tensor, and by substituting the solution into the Klein-Gordon equation and

\footnotetext{
${ }^{15}$ Otherwise the corresponding irrep of the little group (traceless tensor with spatial indices) vanishes, according to a mathematical theorem (see e.g. Chapter 10 in [ [ $]$ ]).
} 
the traceless condition, one obtains the NMG-like equations of motion. The gauge transformation rule for the spin-s gauge field is the derivative of a tensor of the type

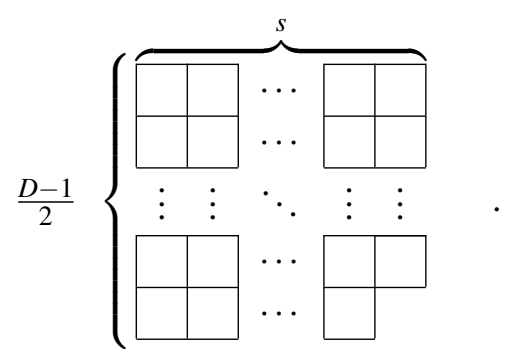

\section{Factorizing the Klein-Gordon Operator}

Just like the 3D cases, in $D=4 k-1$, where $k=1,2, \cdots$, for any tensor $T_{\rho_{1} \cdots \rho_{2 k-1}, \cdots}$ whose symmetry is described by a Young tableau with $2 k-1$ boxes in the first column, its Klein-Gordon equation $\left(\square-m^{2}\right) T_{\rho_{1} \cdots \rho_{2 k-1}, \cdots}=0$ under the divergenceless condition can be written as

$$
\begin{aligned}
& {\left[\frac{1}{(2 k-1) !} \varepsilon_{\mu_{1} \cdots \mu_{2 k-1}}^{\alpha v_{1} \cdots v_{2 k-1}} \partial_{\alpha} \pm m \delta_{\mu_{1} \cdots \mu_{2 k-1}}^{v_{1} \cdots v_{2 k-1}}\right]} \\
& \cdot\left[\frac{1}{(2 k-1) !} \varepsilon_{v_{1} \cdots v_{2 k-1}}^{\beta \rho_{1} \cdots \rho_{2 k-1}} \partial_{\beta} \mp m \delta_{v_{1} \cdots v_{2 k-1}}^{\rho_{1} \cdots \rho_{2 k-1}}\right] T_{\rho_{1} \cdots \rho_{2 k-1}, \cdots}=0,
\end{aligned}
$$

where the Klein-Gordon operator has been factorized into two first-order differential operators. Then by dropping the first operator, one obtains a pair of "generalized $\sqrt{\mathrm{FP}}$ equations" of $T^{16}$

$$
\left[\frac{1}{(2 k-1) !} \varepsilon_{v_{1} \cdots v_{2 k-1}} \beta \rho_{1} \cdots \rho_{2 k-1} \partial_{\beta}-\mu \delta_{v_{1} \cdots v_{2 k-1}}^{\rho_{1} \cdots \rho_{2 k-1}}\right] T_{\rho_{1} \cdots \rho_{2 k-1}, \cdots}=0,
$$

where $\mu= \pm m$. The two equations are interchanged by parity transformation and each carry half of the propagating degrees of freedom of the generalized FP equations of $T$, which is analogous to the 3D situation where the two propagating degrees of freedom split into two helicities.

As for $D=4 k+1$, one can also write down a similar pair of equations, but unfortunately by taking a product of the two first-order differential operators one obtains $-\left(\square+m^{2}\right)$, which is tachyonic, instead of the right Klein-Gordon operator. Therefore, in this situation we don't have a direct generalization of the $\sqrt{\mathrm{FP}}$ equations. ${ }^{17}$ For massive particles in even $D$, there is not any concept in analogy to the helicity in 3D.

We now only focus on the $D=4 k-1$ situation, and we would like to solve the divergenceless condition to boost up the derivatives, in the hope that (4.2) can be converted into a TMG-like model. As already discussed in the previous section, in order to achieve this, we can only use a gauge field whose generalized Einstein tensor, defined as the dual of its generalized Riemann tensor, lives in the same representation as the gauge field itself. Then it is obvious that for spin- $s(s \geq 1)$ the only allowed type of gauge field is represented by a rectangular Young tableau of width $s$ and height

\footnotetext{
${ }^{16}$ Note that the divergenceless and traceless conditions can be derived from either of the two equations.

${ }^{17}$ The spin- 1 models for both $D=4 k-1$ and $D=4 k+1$ were studied in details in [Q] .
} 
$2 k-1$. After substituting the solution to the divergenceless condition $T=G(h)$ into (4.2) one obtains a TMG-like model with a gauge symmetry parameterized by a tensor of the type (B.14). More technical details will be illustrated by the 7D spin-2 example in the next section.

\section{An Example in 7D}

As already explained, for any massive spin $s \geq 1$, the only type of gauge field that is suitable for both NMG and TMG-like models is represented by a rectangular Young tableau of height $2 k-1$ in $4 k-1$ dimensions. In this section, we would like to discuss in details the specific example of $s=2$ and $k=2$, i.e. the type $\square$ in 7D. For both the NMG-like and the TMG-like models, we will construct their equations of motion and integrate them into actions. We will also do an analysis to show that these actions are ghost-free.

To simplify the notation, we use the index with a bar to denote a set of three antisymmetrized indices, e.g. $\bar{\mu}$ stands for the set of indices $\mu_{1} \mu_{2} \mu_{3}$ that are antisymmetrized.

\subsection{The Models}

We start from the tensor field $T_{\bar{\mu}, \bar{v}}$ of the type $\square$. The irrep $\square$ of the little group $\mathrm{SO}(6)$ carries 70 degrees of freedom, so let us first check that after the Lorentz covariantizaton, with the divergenceless and traceless conditions imposed, $T_{\bar{\mu}, \bar{v}}$ carries the same number of degrees of freedom. In the following counting, Young tableaux should be thought as representations of GL(7).

Without any constraints, the number of degrees of freedom carried by $T_{\bar{\mu}, \bar{v}}$ is

$$
\boxminus=490 \text {. }
$$

Now we impose the divergenceless condition $\partial^{\mu_{1}} T_{\bar{\mu}, \bar{v}}=0 .{ }^{18}$ At first sight, this constraint appears to cut the number of degrees of freedom of $\square$, but in fact this corresponds to an excessive cutting, because the double-divergence $\partial^{\mu_{1}} \partial^{\mu_{2}} T_{\bar{\mu}, \bar{v}}$ vanishes by construction, which means the degrees of freedom represented by $\square$ has to be compensated. However, this compensation is again an excessive compensation, because the triple-divergence $\partial^{\mu_{1}} \partial^{\mu_{2}} \partial^{\mu_{3}} T_{\bar{\mu}, \bar{v}}$ vanishes by construction, and we must re-cut the degrees of freedom of $\boxminus$. Therefore, in total

$$
\square-\square+\boxminus=490-210+35=315
$$

degrees of freedom are suppressed by the divergenceless condition.

Then we impose the traceless condition. This means the number of independent components carried by every Young tableau should be reduced by removing its trace. Therefore, the number of degrees of freedom taken away by the traceless condition should be equal to

$$
\square-\square+\square=196-112+21=105 \text {, }
$$

where the first Young tableau stands for the trace of $T_{\bar{\mu}, \bar{v}}$, and the second (third) stands for the trace of the divergence (double-divergence) of $T_{\bar{\mu}, \bar{v}}$.

\footnotetext{
${ }^{18}$ Because $T$ satisfies $T_{\bar{\mu}, \bar{v}}=T_{\bar{v}, \bar{\mu}}$, the divergenceless condition $\partial^{\mu_{1}} T_{\bar{\mu}, \bar{v}}=0$ is equivalent to $\partial^{v_{1}} T_{\bar{\mu}, \bar{v}}=0$.
} 
In the end, we find that $T_{\bar{\mu}, \bar{v}}$ under both constraints indeed carries 70 degrees of freedom:

$$
490-315-105=70 \text {. }
$$

Then we can write down the generalized FP equations:

$$
\left(\square-m^{2}\right) T_{\bar{\mu}, \bar{v}}=0, \partial^{\mu_{1}} T_{\bar{\mu}, \bar{v}}=0, \eta^{\mu_{1} v_{1}} T_{\bar{\mu}, \bar{v}}=0,
$$

and the generalized $\sqrt{\mathrm{FP}}$ equation:

$$
\left(\frac{1}{6} \varepsilon_{\bar{\mu}}^{\alpha \bar{\rho}} \partial_{\alpha}-\mu \delta_{\bar{\mu}}^{\bar{\rho}}\right) T_{\bar{\rho}, \bar{v}}=0,
$$

where $\mu= \pm m$, and each sign corresponds to an equation describing 35 propagating degrees of freedom. Note that the divergenceless and traceless condtions can be derived from (5.6).

In the next step, one can solve the divergenceless condition by

$$
T_{\bar{\mu}, \bar{v}}=G_{\bar{\mu}, \bar{v}}(h) \equiv \varepsilon_{\bar{\mu}}^{\alpha \bar{\rho}} \varepsilon_{\bar{v}}^{\beta \bar{\sigma}} \partial_{\alpha} \partial_{\beta} h_{\bar{\rho}, \bar{\sigma}},
$$

where the gauge field $h$ and the generalized Einstein tensor $G(h)$ also belong to the type $\square$. Thus one may boost up the derivatives to derive the NMG-like equations of motion

$$
\left(\square-m^{2}\right) G_{\bar{\mu}, \bar{v}}(h)=0, \quad \eta^{\mu_{1} v_{1}} G_{\bar{\mu}, \bar{v}}(h)=0,
$$

and the TMG-like equation of motion

$$
\left(\frac{1}{6} \varepsilon_{\bar{\mu}}^{\alpha \bar{\rho}} \partial_{\alpha}-\mu \delta_{\bar{\mu}}^{\bar{\rho}}\right) G_{\bar{\rho}, \bar{v}}(h)=0,
$$

from which one can also derive $\eta^{\mu_{1} v_{1}} G_{\bar{\mu}, \bar{v}}(h)=0$.

Analogous to $3 \mathrm{D}$, we can define the generalized Cotton tensor ${ }^{19}$

$$
C_{\bar{\mu}, \bar{v}}(h)=\mathscr{Y}_{[3,3]}\left[\mathcal{E}_{\bar{\mu}}^{\alpha \bar{\rho}} \partial_{\alpha} G_{\bar{\rho}, \bar{v}}(h)\right],
$$

which is both divergenceless and traceless, and we can use it to simplify the construction of both the NMG-like and the TMG-like actions.

The NMG-like action reads

$$
S_{7 \mathrm{D} \mathrm{NMG} \mathrm{spin-2}}=\int d^{7} x\left\{\frac{1}{72} h^{\bar{\mu}, \bar{v}} \varepsilon_{\bar{\mu}}^{\alpha \bar{\rho}} \partial_{\alpha} C_{\bar{\rho}, \bar{v}}(h)-\frac{1}{2} m^{2} h^{\bar{\mu}, \bar{v}} G_{\bar{\mu}, \bar{v}}(h)\right\},
$$

from which one can derive $\mathrm{e}^{20}$

$$
\frac{1}{36} \varepsilon_{\bar{\mu}}^{\alpha \bar{\rho}} \partial_{\alpha} C_{\bar{\rho}, \bar{v}}(h)-m^{2} G_{\bar{\mu}, \bar{v}}(h)=0 .
$$

\footnotetext{
${ }^{19}$ Note that off-shell we need the Young symmetrizer, otherwise $C_{\bar{\mu}, \bar{v}}(h)$ does not satisfy the symmetry However, on-shell by using the traceless condition of $G_{\bar{\mu}, \bar{v}}(h)$ one can prove that dropping the Young symmetrizer gives an equivalent formula.

${ }^{20}$ Because the generalized Cotton tensor is both divergenceless and traceless, one can prove that the first term in this equation of motion, without being projected by a Young symmetrizer, already satisfies the symmetry
} 
By taking the trace of this equation one obtains

$$
\eta^{\mu_{1} v_{1}} G_{\bar{\mu}, \bar{v}}(h)=0,
$$

and substituting it back into (5.12) gives

$$
\left(\square-m^{2}\right) G_{\bar{\mu}, \bar{v}}(h)=0 .
$$

Thus (5.8) is derived.

The TMG-like action reads

$$
S_{7 \mathrm{D} \text { TMG spin-2 }}=\int d^{7} x\left\{\frac{1}{12} h^{\bar{\mu}, \bar{v}} C_{\bar{\mu}, \bar{v}}(h)-\frac{1}{2} \mu h^{\bar{\mu}, \bar{v}} G_{\bar{\mu}, \bar{v}}(h)\right\},
$$

from which one can derive

$$
\frac{1}{6} C_{\bar{\mu}, \bar{v}}(h)-\mu G_{\bar{\mu}, \bar{v}}(h)=0,
$$

and the trace of this equation gives the traceless condition

$$
\eta^{\mu_{1} v_{1}} G_{\bar{\mu}, \bar{v}}(h)=0 .
$$

Using this condition one can prove that (5.J) and (5.9) are equivalent.

\subsection{Canonical Analysis}

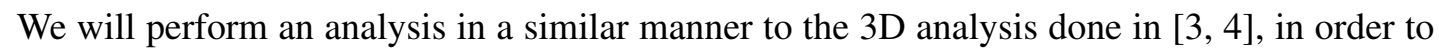
show that the actions (5. 1 ) and $(5 \cdot 5)$ ) indeed describe 70 and 35 (for each choice of $\mu$ ) propagating degrees of freedom, respectively, which are ghost-free.

Before the analysis, we first count the number of degrees of freedom carried by the gauge field $h$. The gauge field $h$ of the type $\square$, which is counted as the 490 representation of GL(7), transforms under the gauge transformations

$$
\delta h_{\mu_{1} \mu_{2} \mu_{3}, v_{1} v_{2} v_{3}}=\mathscr{Y}_{[3,3]}\left(\partial_{\left[v_{3} \mid\right.} \xi_{\left.\mu_{1} \mu_{2} \mu_{3}, v_{1} v_{2}\right]}\right)
$$

where $\xi$ is of the type $\square$, which has 490 degrees of freedom. We should be careful with the counting because $\xi$ has its own gauge symmetry

$$
\delta \xi_{\mu_{1} \mu_{2} \mu_{3}, v_{1} v_{2}}=\mathscr{Y}_{[3,2]}\left(\partial_{\left[v_{2} \mid\right.} \zeta_{\left.\mu_{1} \mu_{2} \mu_{3}, \mid v_{1}\right]}\right)
$$

where $\zeta$ is of the type $\square$ with 210 degrees of freedom. Moreover $\zeta$ has also by itself a gauge symmetry

$$
\delta \zeta_{\mu_{1} \mu_{2} \mu_{3}, v_{1}}=\mathscr{Y}_{[3,1]}\left(\partial_{v_{1}} \lambda_{\mu_{1} \mu_{2} \mu_{3}}\right)
$$

where $\lambda$ is an antisymmetric tensor and has 35 degrees of freedom. Therefore, to summarize the above counting, we expect that after a gauge-fixing $h$ should carry

$$
490-490+210-35=175
$$

degrees of freedom. 
Now we split the indices into temporal and spatial components like $\mu=(0, i), i=1, \cdots, 6$, and impose the gauge-fixing condition ${ }^{21}$

$$
\partial^{i} h_{i \mu_{2} \mu_{3}, v_{1} v_{2} v_{3}}=0 .
$$

Under this condition, we parameterize $h$ in terms of the independent components $(a, b, c, d, e)$ as follows: ${ }^{22}$

$$
\begin{aligned}
h_{0 i_{2} i_{3}, 0 j_{2} j_{3}}= & a_{i_{2} i_{3}, j_{2} j_{3}}, \\
h_{0 i_{2} i_{3}, j_{1} j_{2} j_{3}}= & \varepsilon_{j_{1} j_{2} j_{3}}{ }^{{ }_{1} k_{2} k_{3}} \partial_{k_{1}} b_{k_{2} k_{3}, i_{2} i_{3}}+\left\{\left(\delta_{i_{3} j_{3}}-\frac{\partial_{i_{3}} \partial_{j_{3}}}{\nabla^{2}}\right) c_{j_{1} j_{2}, i_{2}}\right. \\
& \left.+\left(\delta_{i_{2} j_{2}} \delta_{i_{3} j_{3}}-\frac{\partial_{i_{2}} \partial_{j_{2}}}{\nabla^{2}} \delta_{i_{3} j_{3}}-\delta_{i_{2} j_{2}} \frac{\partial_{i_{3}} \partial_{j_{3}}}{\nabla^{2}}\right) d_{j_{1}}\right\}_{\text {a.s. }}, \\
h_{i_{1} i_{2} i_{3}, j_{1} j_{2} j_{3}}= & \varepsilon_{i_{1} i_{2} i_{3}}{ }^{k_{1} k_{2} k_{3}} \varepsilon_{j_{1} j_{2} j_{3}}{ }^{l_{1} l_{2} l_{3}} \partial_{k_{1}} \partial_{l_{1}} e_{k_{2} k_{3}, l_{2} l_{3}},
\end{aligned}
$$

where the properties of these components are listed below.

\begin{tabular}{|c|c|c|c|c|}
\hline Components & Symmetry & divergence & trace & degrees \\
\hline \hline$a_{. . . .}$ & $\square$ & -less & -ful & 50 \\
\hline$b_{. . . .}$ & $\square$ & -less & -less & 35 \\
\hline$c_{. . . .}$ & $\square$ & -less & -less & 35 \\
\hline$d_{.}$ & $\square$ & -less & & 5 \\
\hline$e_{. . . .}$ & $\square$ & -less & -ful & 50 \\
\hline
\end{tabular}

As shown in the last column of this table, the total number of degrees of freedom sums up to 175 , which is consistent with (5.2I).

In the next step, we substitute (5.231) into (5.II) and (5.5]). Then we separate the trace of $a_{i_{2} i_{3}, j_{2} j_{3}}$ from its traceless part:

$$
a_{i_{2} i_{3}, j_{2} j_{3}}=\hat{a}_{i_{2} i_{3}, j_{2} j_{3}}+\left\{\left(\eta_{i_{2} j_{2}}-\frac{\partial_{i_{2}} \partial_{j_{2}}}{\nabla^{2}}\right) \bar{a}_{i_{3}, j_{3}}+\left(\eta_{i_{2} j_{2}} \eta_{i_{3} j_{3}}-\frac{\partial_{i_{2}} \partial_{j_{2}}}{\nabla^{2}} \eta_{i_{3} j_{3}}-\eta_{i_{2} j_{2}} \frac{\partial_{i_{3}} \partial_{j_{3}}}{\nabla^{2}}\right) a\right\}_{\text {a.s. }},
$$

where $\hat{a}_{i_{2} i_{3}, j_{2} j_{3}}$ and $\bar{a}_{i_{3}, j_{3}}$ are traceless and carry 35 and 14 degrees of freedom, which represent the traceless and the trace parts of $a_{i_{2} i_{3}, j_{2} j_{3}}$, respectively, and $a$ carrying one degree of freedom represents the double trace part. We also split $e_{i_{2} i_{3}, j_{2} j_{3}}$ in the same way.

In the resulting NMG-like action, we further do a field redefinition: ${ }^{23}$

$$
\hat{a}_{i_{2} i_{3}, j_{2} j_{3}}=\tilde{a}_{i_{2} i_{3}, j_{2} j_{3}}-4 \square \hat{e}_{i_{2} i_{3}, j_{2} j_{3}}+8 m^{2} \hat{e}_{i_{2} i_{3}, j_{2} j_{3}} .
$$

\footnotetext{
${ }^{21}$ One also has to gauge-fix $\partial^{i_{2}} \xi_{i_{2} \mu_{3}, v_{1} v_{2} v_{3}}=0$ and $\partial^{i_{3}} \zeta_{i_{3}, v_{1} v_{2} v_{3}}=0$ to show that the gauge parameters indeed cannot carry any propagating degrees of freedom.

${ }^{22}$ The notation \{\}$_{\text {a.s }}$ stands for antisymmetrizing all indices within the curly bracket that have the same Latin letter. For instance, $\left\{T_{i_{2} i_{3} j_{1} j_{2} j_{3}}\right\}_{\text {a.s. }}=T_{\left[i_{2} i_{3}\right]\left[j_{1} j_{2} j_{3}\right]} . \varepsilon_{i j k l m n} \equiv \varepsilon_{0 i j k l m n} . \nabla^{2} \equiv \partial^{i} \partial_{i}$. Note that we permit space non-locality, since this does not affect the canonical structure.

${ }^{23}$ In the context of this analysis, the $\square$ operator is simply understood as a notation for $-\left(\partial_{0}\right)^{2}+\nabla^{2}$, rather than being interpreted as a Lorentz-invariant object.
} 
In the end we obtain

$$
\begin{array}{rl}
S_{7 \mathrm{D} \mathrm{NMG} \mathrm{spin-2}}=\int d^{7} & x\left\{(3 !)^{4} b^{i_{2} i_{3}, j_{2} j_{3}}\left(\nabla^{2}\right)^{2}\left(\square-m^{2}\right) b_{i_{2} i_{3}, j_{2} j_{3}}\right. \\
& +4(3 !)^{4} m^{2} \hat{e}^{i_{2} i_{3}, j_{2} j_{3}}\left(\nabla^{2}\right)^{2}\left(\square-m^{2}\right) \hat{e}_{i_{2} i_{3}, j_{2} j_{3}} \\
& +\frac{1}{16}(3 !)^{4} \tilde{a}^{i_{2} i_{3}, j_{2} j_{3}}\left(\nabla^{2}\right)^{2} \tilde{a}_{i_{2} i_{3}, j_{2} j_{3}}-\frac{3}{4}(3 !)^{4} m^{2} \bar{a}^{i_{3}, j_{3}}\left(\nabla^{2}\right)^{2} \bar{e}_{i_{3}, j_{3}} \\
& -10(3 !)^{4} m^{2} a\left(\nabla^{2}\right)^{2} e-\frac{3}{10}(5 !) m^{2} c^{j_{1} j_{2}, i_{2}} \nabla^{2} c_{j_{1} j_{2}, i_{2}} \\
& \left.+\frac{9}{2}(2 ! 4 !) m^{2} d^{j_{1}} \nabla^{2} d_{j_{1}}\right\} .
\end{array}
$$

We see that the first and second terms each represent 35 propagating degrees of freedom, both with the right sign, and the rest part decouples and does not propagate. Thus, this action describes 70 massive physical degrees of freedom.

In the resulting TMG-like action, we do the following field redefinitions:

$$
\hat{a}_{i_{2} i_{3}, j_{2} j_{3}}=\tilde{a}_{i_{2} i_{3}, j_{2} j_{3}}-\frac{2}{\mu} \square b_{i_{2} i_{3}, j_{2} j_{3}}, \quad \hat{e}_{i_{2} i_{3}, j_{2} j_{3}}=\tilde{e}_{i_{2} i_{3}, j_{2} j_{3}}-\frac{1}{2 \mu} b_{i_{2} i_{3}, j_{2} j_{3}},
$$

which gives

$$
\begin{array}{rl}
S_{7 \mathrm{D} T M G \text { spin- } 2}=\int d^{7} & x\left\{\frac{1}{\mu}(3 !)^{4} b^{i_{2} i_{3}, j_{2} j_{3}}\left(\nabla^{2}\right)^{2}\left(\square-\mu^{2}\right) b_{i_{2} i_{3}, j_{2} j_{3}}\right. \\
& -(3 !)^{4} \mu \tilde{a}^{i_{2} i_{3}, j_{2} j_{3}}\left(\nabla^{2}\right)^{2} \tilde{e}_{i_{2} i_{3}, j_{2} j_{3}}-\frac{3}{4}(3 !)^{4} \mu \bar{a}^{i_{3}, j_{3}}\left(\nabla^{2}\right)^{2} \bar{B}_{i_{3}, j_{3}} \\
& -10(3 !)^{4} \mu a\left(\nabla^{2}\right)^{2} e-\frac{3}{10}(5 !) \mu c^{j_{1} j_{2}, i_{2}} \nabla^{2} c_{j_{1} j_{2}, i_{2}} \\
+ & \left.\frac{9}{2}(2 ! 4 !) \mu d^{j_{1}} \nabla^{2} d_{j_{1}}\right\} .
\end{array}
$$

The first term contains 35 propagating degrees of freedom and the rest terms are all auxiliary, and by properly choosing an overall sign, this action is ghost-free.

\section{Conclusions}

We have discussed the possibility to extend 3D NMG/TMG-like models to higher dimensions. The conclusion is that only when the dual of the Riemann tensor lives in the same representation as the gauge field, and the corresponding Young tableau has no more than $D-1$ boxes in its first two columns, can we have a chance to extend the NMG-like models. Furthermore, to extend the TMG-like models, additionally the number of spacetime dimensions has to be $4 k-1, k=1,2, \cdots$, and the corresponding Young tableau has to be a rectangle of height $2 k-1$.

We have explicitly shown the example $\square$ in 7D. The actions (5.TI) and (5.5I) look almost the same as the 3D linearized NMG and TMG actions in [B]], except for the bars on the indices and some coefficients. The canonical analysis is also similar. Furthermore, in [ए]] we also discussed that the mass term, i.e. the second order derivative term shared by (5.J1) and (5.15), can be reformulated at the linearized level in a similar way to the 3D Chern-Simons formalism in [प], प2]. We hope 
these similarities may give us further inspiration on how to introduce interactions into the higher dimensional models.

Acknowledgements This paper is based on the author's presentation in the XVIII European Workshop on String Theory. The author would like to thank the organizers of the 2012 Corfu Summer Institute for giving him the opportunity to present his work ([U, 미] in collaboration with E.A. Bergshoeff, M. Kovacevic, J. Rosseel and P.K. Townsend). The author would also like to thank Eric A. Bergshoeff for helpful comments on this paper. The work of the author is supported by the Ubbo Emmius Programme administered by the Graduate School of Science, University of Groningen. Some calculations in the paper were done by the software Cadabra [[13], [4]].

\section{References}

[1] E. A. Bergshoeff, O. Hohm, and P. K. Townsend, "Massive Gravity in Three Dimensions," Phys.Rev.Lett. 102 (2009) 201301, arXiv:0901.1766 [hep-th].

[2] S. Deser, R. Jackiw, and S. Templeton, "Topologically Massive Gauge Theories," Annals Phys. 140 1982) 372-411.

[3] E. A. Bergshoeff, O. Hohm, and P. K. Townsend, "On Higher Derivatives in 3D Gravity and Higher Spin Gauge Theories," Annals Phys. 325 (2010) 1118-1134, arXiv:0911.3061 [hep-th].

[4] E. A. Bergshoeff, M. Kovacevic, J. Rosseel, P. K. Townsend, and Y. Yin, "A spin-4 analog of 3D massive gravity," Class.Quant.Grav. 28 (2011) 245007, arXiv:1109.0382 [hep-th].

[5] D. Francia and C. Hull, "Higher-spin gauge fields and duality," arXiv:hep-th/0501236 thep-th.

[6] X. Bekaert and N. Boulanger, "Tensor gauge fields in arbitrary representations of GL(D,R): Duality and Poincare lemma," Commun.Math.Phys. 245 (2004) 27-67, arXiv: hep-th/0208058 thep-th.

[7] E. A. Bergshoeff, J. Fernandez-Melgarejo, J. Rosseel, and P. K. Townsend, "On 'New Massive' 4D Gravity," WHEP 1204(2012) 070, arXiv: 1202.1501 [hep-th].

[8] M. Hamermesh, Group theory and its application to physical problems. Addison-Wesley series in physics. Addison-Wesley Pub. Co., 1962.

[9] P. Townsend, K. Pilch, and P. van Nieuwenhuizen, "Selfduality in Odd Dimensions," Phys.Lett. B136 (1984) 38.

[10] E. A. Bergshoeff, M. Kovacevic, J. Rosseel, and Y. Yin, "On Topologically Massive Spin-2 Gauge Theories beyond Three Dimensions," DHEP 1210 (2012) 055, arXiv:1207.0192 hep-th].

[11] A. Achucarro and P. Townsend, "A Chern-Simons Action for Three-Dimensional anti-De Sitter Supergravity Theories," Phys.Lett. B180(1986) 89.

[12] E. Witten, “(2+1)-Dimensional Gravity as an Exactly Soluble System," Nucl.Phys. B311 (1988) 46.

[13] K. Peeters, "Cadabra: a field-theory motivated symbolic computer algebra system,' Computer Physics Communications 176 (Apr., 2007) 550-558, arXiv: cS/0608005.

[14] K. Peeters, "Introducing Cadabra: A Symbolic computer algebra system for field theory problems," arXiv:hep-th/0701238 [HEP-TH]. 